\title{
Min-Bias At The Tevatron
}

\author{
Rick Field ${ }^{1}$ \\ (for the CDF Collaboration)
}

Department of Physics, University of Florida, Gainesville, Florida, 32611, USA

\begin{abstract}
Tevatron data on minimum bias collisions are examined and the modeling of this process is discussed. Extrapolations to the LHC are studied.
\end{abstract}

Keywords: QCD, Min-Bas, Jets, Hadron Collider.

PACS: 12.38.-t, 12.38.Bx, 12.38.Qk

As illustrated in Fig. 1, the total proton-antiproton cross section is the sum of the elastic and inelastic components, $\sigma_{\mathrm{tot}}=\sigma_{\mathrm{EL}}+\sigma_{\mathrm{IN}}$. The inelastic cross section consists of three terms; single diffraction, double-diffraction, and everything else (referred to as the "hard core"), $\sigma_{\mathrm{IN}}=\sigma_{\mathrm{SD}}+\sigma_{\mathrm{DD}}+\sigma_{\mathrm{HC}}$. For elastic scattering neither of the beam particles breaks apart (i.e. color singlet exchange). For single and double diffraction one or both of the beam particles are excited into a high mass color singlet state (i.e. $\mathrm{N}^{*}$ states) which then decays. Single and double diffraction also corresponds to color singlet exchange between the beam hadrons. When color is exchanged the outgoing remnants are no longer color singlets and one has a separation of color resulting in a multitude of quark-antiquark pairs being pulled out of the vacuum. The "hard core" component, $\sigma_{\mathrm{HC}}$, involves color exchange and the separation of color. However, the "hard core" contribution has both a "soft" and "hard" component. Most of the time the color exchange between partons in the beam hadrons occurs through a soft interaction (i.e. no high transverse momentum) and the two beam hadrons "ooze" through each other producing lots of soft particles with a uniform distribution in rapidity and many particles flying down the beam pipe. Occasionally there is a hard scattering among the constituent partons producing outgoing particles and "jets" with high transverse momentum.

Experimentally it is difficult to separate $\sigma_{\mathrm{HC}}$ from $\sigma_{\mathrm{DD}}$. At $1.8 \mathrm{TeV}$ (CDF Run 1) the total proton-antiproton cross section is about $78 \mathrm{mb}$ and the elastic cross section is about $18 \mathrm{mb}$ [1,2]. Single diffraction makes up about $9 \mathrm{mb}$ of the $60 \mathrm{mb}$ inelastic cross section and $\sigma_{\mathrm{HC}}+\sigma_{\mathrm{DD}} \approx 51 \mathrm{mb}$, with double diffraction in the range $4<\sigma_{\mathrm{DD}}<7$ $\mathrm{mb}$ [3]. Hadron-hadron collider experiments do not usually collect every event that occurs. One selects (i.e. "triggers" on) certain events to store onto tape. Minimum bias (i.e. "min-bias") is a generic term which refers to events that are selected with a "loose" trigger that accepts a large fraction of the inelastic cross section. All triggers produce some bias and the term "min-bias" is meaningless until one specifies the precise trigger used to collect the data. The CDF "min-bias" trigger consists of

\footnotetext{
${ }^{1}$ To appear in the Proceedings of the Colliders to Cosmic Ray Conference (C2CR07), Feb. 25 - March 1, Lake Tahoe, CA.
} 
requiring at least one charged particle in the forward region $3.2<\eta<5.9$ and simultaneously at least one charged particle in the backward region $-5.9<\eta<-3.2$. Monte-Carlo studies show that the CDF "min-bias" collects most of the $\sigma_{\mathrm{HC}}$ contribution plus small amounts of single and double diffraction.

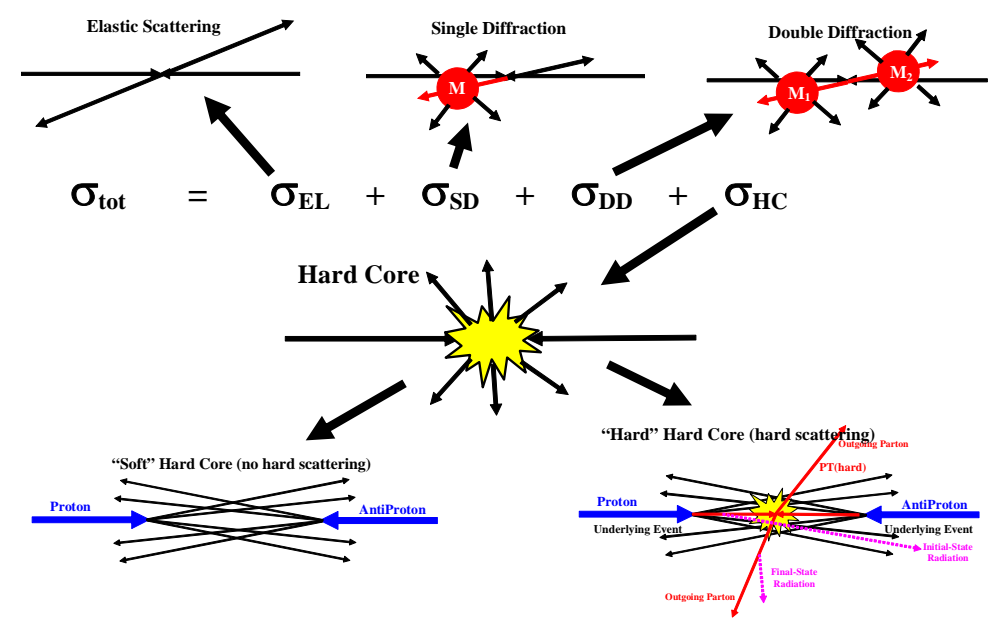

FIGURE 1. Shows the various components of the proton-antiproton total cross section, $\sigma_{\text {tot }}$. The total cross section is the sum of the elastic and inelastic components, $\sigma_{\mathrm{tot}}=\sigma_{\mathrm{EL}}+\sigma_{\mathrm{IN}}$. The inelastic cross section consists of three terms; single diffraction, double-diffraction, and everything else (referred to as the "hard core"), $\sigma_{\mathrm{IN}}=\sigma_{\mathrm{SD}}+\sigma_{\mathrm{DD}}+\sigma_{\mathrm{HC}}$. The "hard core", $\sigma_{\mathrm{HC}}$, contribution has both a "soft" and "hard" component (i.e. "soft" hard core and "hard" hard core).

Fig. 2 shows that "min-bias" collisions in Run 1 at CDF produce about 4 charged particles per unit pseudorapidity at $\eta=0$ [4-5]. This corresponds to about 0.67 charged particles per unit $\eta-\phi$ at $\eta=0$ (i.e. $\approx 4 / 2 \pi)$. In Run 2 at CDF, we measure only a fraction of the charged particles produced in the collision since we are restricted to the range $\mathrm{p}_{\mathrm{T}}>0.5 \mathrm{GeV} / \mathrm{c}$ and $|\eta|<1$, where the central-outer-tracker (i.e. COT) is very efficient. For "min-bias" collisions at $1.96 \mathrm{TeV}$ there are about 0.25 charged particles per unit $\eta-\phi$ for the range $\mathrm{p}_{\mathrm{T}}>0.5 \mathrm{GeV} / \mathrm{c}$ and $|\eta|<1$.
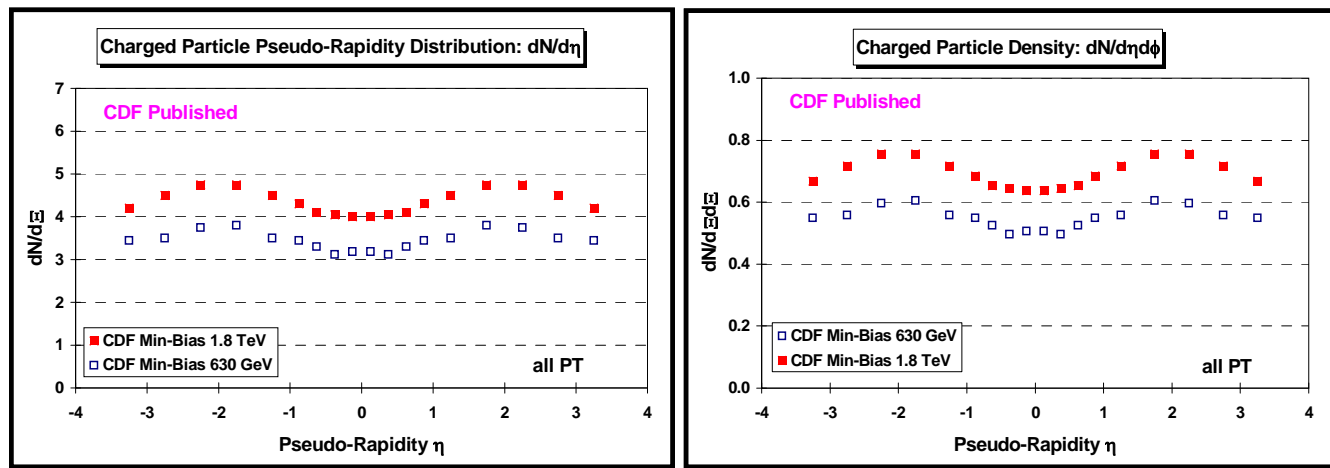

FIGURE 2. (left) Shows the CDF Run 1 "min-bias" data on the pseudorapidity distribution, $\mathrm{dN} / \mathrm{d} \eta$, of charged particles (all $\mathrm{p}_{\mathrm{T}}$ ) at $1.8 \mathrm{TeV}$ and $630 \mathrm{GeV}$. At $1.8 \mathrm{TeV}$ the number of charged particles per unit 
pseudorapidity at $\eta=0$ is about 4 . (right) Shows the density of charged particles in $\eta-\phi$ space, $d N / d \eta d \phi$, at $1.8 \mathrm{TeV}$ and $630 \mathrm{GeV}$. At $1.8 \mathrm{TeV}$ the number of charged particles per unit $\eta-\phi$ at $\eta=0$ is about 0.67 .
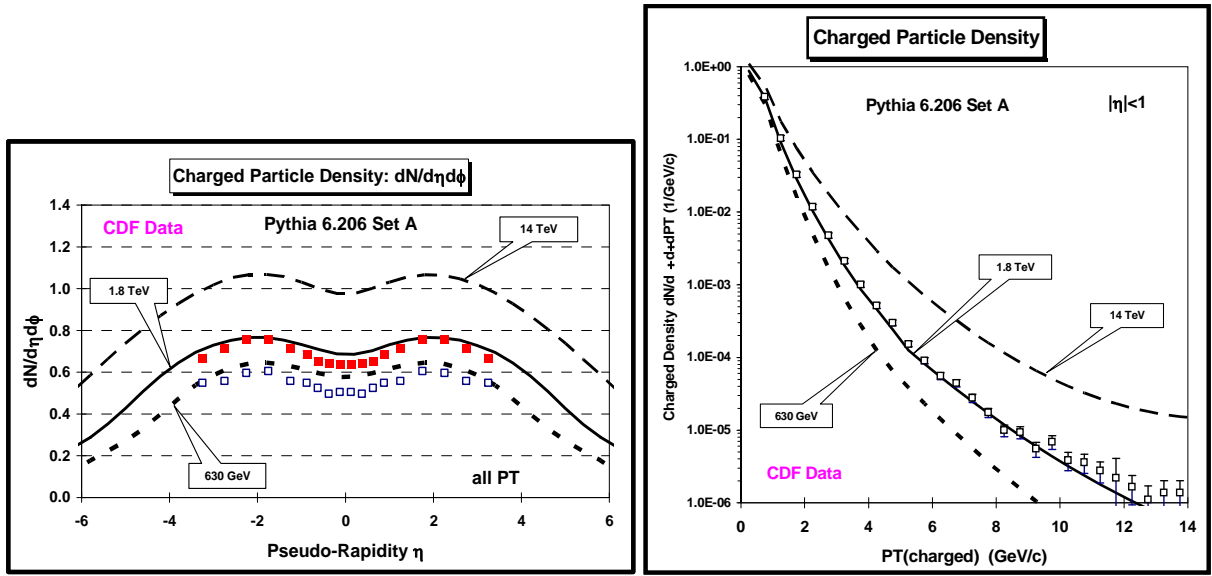

FIGURE 3. (left) Shows the CDF Run1 "min-bias" data on the charged particle density, $\mathrm{dN} / \mathrm{d} \eta \mathrm{d} \phi$, for all $\mathrm{p}_{\mathrm{T}}$ at $630 \mathrm{GeV}$ and $1.8 \mathrm{TeV}$. (right) Shows the CDF Run1 "min-bias" data on the $\mathrm{p}_{\mathrm{T}}$ dependence of charged particle density, $\mathrm{dN} / \mathrm{d} \eta \mathrm{d}_{\mathrm{d}} \mathrm{dp}_{\mathrm{T}}$, for $|\eta|<1$ at $1.8 \mathrm{TeV}$. Also shown are the predictions of PYTHIA Tune A $630 \mathrm{GeV}, 1.8 \mathrm{TeV}$, and $14 \mathrm{TeV}$.

Fig. 3 shows a comparison of the CDF Run 1 data [4-5] on the $\eta$ dependence and the $\mathrm{p}_{\mathrm{T}}$ dependence of the charged particle density for "min-bias" collisions with PYTHIA Tune A [6,7]. PYTHIA Tune A is the first model for "min-bias" collisions that comes anywhere close to describing the CDF data. HERWIG [8] has a "soft" min-bias model based on "jet" fragmentation which describes the charged particle $\mathrm{dN} / \mathrm{d} \eta$ distribution (i.e. 4 charged particles per unit $\eta$ ), however, the model contains no hard collisions which results in a charged particle $\mathrm{p}_{\mathrm{T}}$ distribution that falls off much more rapidly than the data in Fig. 3. It is difficult to find a model with the correct mixture of "hard" and "soft" collisions observed in the hard core inelastic cross section. The 2-to-2 parton-parton perturbative cross section diverges at low $\mathrm{p}_{\mathrm{T}}$. To describe "min-bias" collision one must keep the low $\mathrm{p}_{\mathrm{T}}$ 2-to-2 parton-parton scattering cross sections from diverging. PYTHIA does this by multiplying the 2-to-2 cross section by the factor $\hat{p}_{T}^{4} /\left(\hat{p}_{T}^{2}+\hat{p}_{T 0}^{2}\right)^{2}$, where $\hat{p}_{T}$ is the transverse momentum of the 2 to-2 parton-parton scattering and $\hat{p}_{T 0}$ is a cut-off parameter that is around 1.5-2.0 $\mathrm{GeV} / \mathrm{c}$ (for PYTHIA Tune A $\hat{p}_{T 0}=2.0 \mathrm{GeV} / \mathrm{c}$ at $1.8 \mathrm{TeV}$ ). This factor regulates the 2-to-2 divergences and goes to one for large transverse momentum, thus reproducing the perturbative prediction. Of course this is done at the expense of introducing a new parameter $\hat{p}_{T 0}$. However, PYTHIA uses this same factor with the same parameter to regulate the additional 2-to-2 parton-parton scattering that may occur during the same proton-antiproton collision (i.e. multiple parton interactions). Varying the cut-off $\hat{p}_{T 0}$ changes the multiple parton interaction cross section which determines the number of multiple parton interactions per collision. PYTHIA Tune A was not tuned to fit "min-bias" data. It was tuned to describe the CDF Run 1 "underlying event" data in high $\mathrm{p}_{\mathrm{T}}$ "chgjet" production [9]. The cut-off parameter $\hat{p}_{T 0}$ was chosen to give the 
correct amount of multiple parton interactions in "hard" scattering collisions. It is amazing that Tune A also does a fairly good job in describing "min-bias" collisions.

We would like to know how much of the hard core cross section is "hard" and to know how "jetty" it is. In Run 2 at CDF, we examine the jet structure in "min-bias" collisions by studying correlations among the charged particles. We examine correlations in azimuthal angle $\Delta \phi$ relative to the direction of the highest $\mathrm{p}_{\mathrm{T}}$ charged particle $\left(\mathrm{p}_{\mathrm{T}}>0.5 \mathrm{GeV} / \mathrm{c},|\eta|<1\right)$ in the event, PTmax. The $\Delta \phi$ dependence of the associated density of charged particles, $\mathrm{dN} / \mathrm{d} \eta \mathrm{d} \phi$, for charged particles with $\mathrm{p}_{\mathrm{T}}>0.5$ GeV/c and $|\eta|<1$ (not including PTmax) are plotted relative to the direction of PTmax which is rotated to $\Delta \phi=180^{\circ}$. The associated density is a measure of the particles accompanying the maximum $\mathrm{p}_{\mathrm{T}}$ charged particle. Fig. 4 shows the CDF Run 2 data on the $\Delta \phi$ dependence of the "associated" density of charged particles relative to the direction of PTmax for PTmax $>0.5 \mathrm{GeV} / \mathrm{c},>1.0 \mathrm{GeV} / \mathrm{c}$, and $>2.0 \mathrm{GeV} / \mathrm{c}$ for "minbias" collisions at $1.96 \mathrm{TeV}$. The data show a very strong correlation among the charged particles indicating "jet structure" in "min-bias" collisions even for momentums as low as $1 \mathrm{GeV} / \mathrm{c}$. In fact, the density of charged particles associated with PTmax in the "toward" region is larger than the average density of charged particles in "min-bias" collisions. This means that it is more probable to find a charged particle accompanying PTmax than it is to find one charged particle in a typical "minbias" collision!

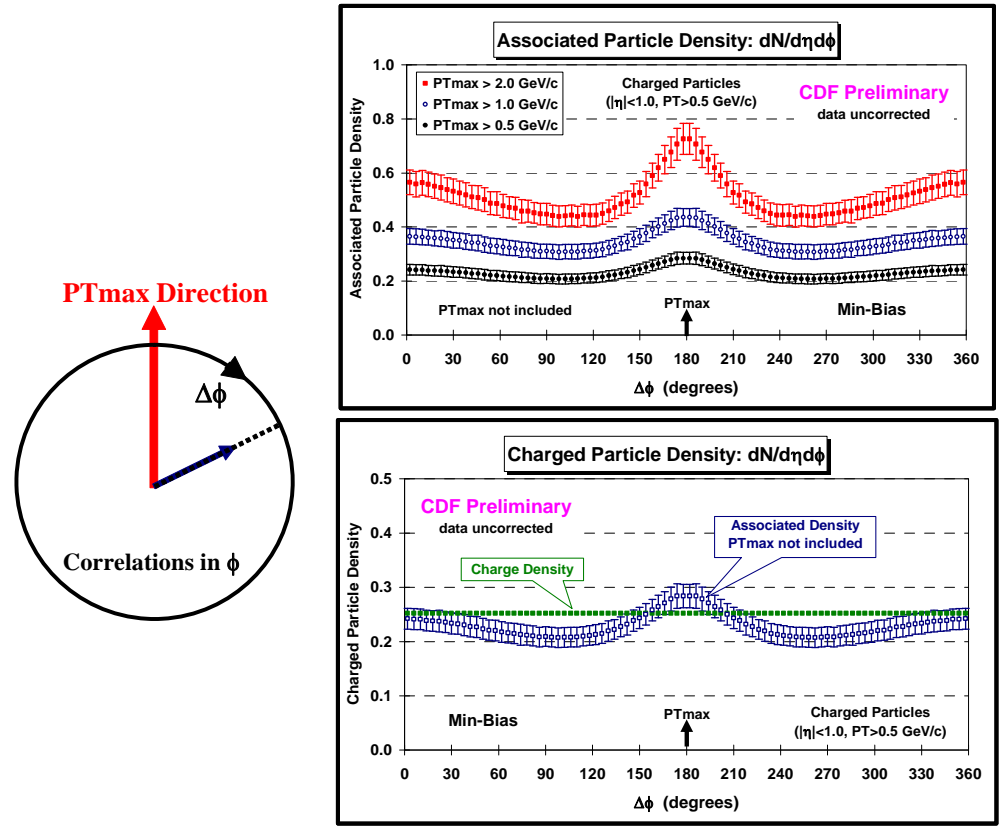

FIGURE 4. (top) CDF Run 2 data on the $\Delta \phi$ dependence of the "associated" density of charged particles, $\mathrm{dN} / \mathrm{d} \eta \mathrm{d} \phi$, with $\mathrm{p}_{\mathrm{T}}>0.5 \mathrm{GeV} / \mathrm{c}$ and $|\eta|<1$ (not including PTmax) relative to the direction of PTmax (rotated to $180^{\circ}$ ) with PT max $>0.5 \mathrm{GeV} / \mathrm{c}$, PTmax $>1.0 \mathrm{GeV} / \mathrm{c}$, and PTmax $>2.0 \mathrm{GeV} / \mathrm{c}$ for " $\mathrm{min}$ bias" collisions at $1.96 \mathrm{TeV}$. (bottom) Run 2 data on the $\Delta \phi$ dependence of the "associated" density of charged particles, $\mathrm{dN} / \mathrm{d} \eta \mathrm{d} \phi$, for with $\mathrm{p}_{\mathrm{T}}>0.5 \mathrm{GeV} / \mathrm{c}$ and $|\eta|<1$ (not including PTmax) relative to the direction of PTmax (rotated to $180^{\circ}$ ) compared with the average density of charged particles, $\mathrm{dN} / \mathrm{d} \eta \mathrm{d} \phi \approx 0.25$, with $\mathrm{p}_{\mathrm{T}}>0.5 \mathrm{GeV} / \mathrm{c}$ and $|\eta|<1$ for "min-bias" collisions at $1.96 \mathrm{TeV}$. 
Fig. 5 shows a comparison of CDF data on the "min-bias" associated charged particle density with PYTHIA Tune A. The plot shows (uncorrected) data compared with PYTHIA Tune A after detector simulation (i.e. CDFSIM). Tune A does a fairly good job describing the this associated density, although Tune A is a bit more "jetty" than the data. Figs. 4 and 5 show the "birth" of jet\#1 in "min-bias" collisions. One can also see the "birth" of jet\#2, which results in the rise in the number density in the region $180^{\circ}$ from jet\#1 (i.e. the "away" region). Fig. 5 also shows a rapid increase in the activity in the "transverse" region as PTmax increases to $2.0 \mathrm{GeV} / \mathrm{c}$. The "transverse" region is very sensitive to the modeling of the "underlying event" (i.e. beam-beam remnants and multiple parton interactions) and Tune A also describes this region fairly well.
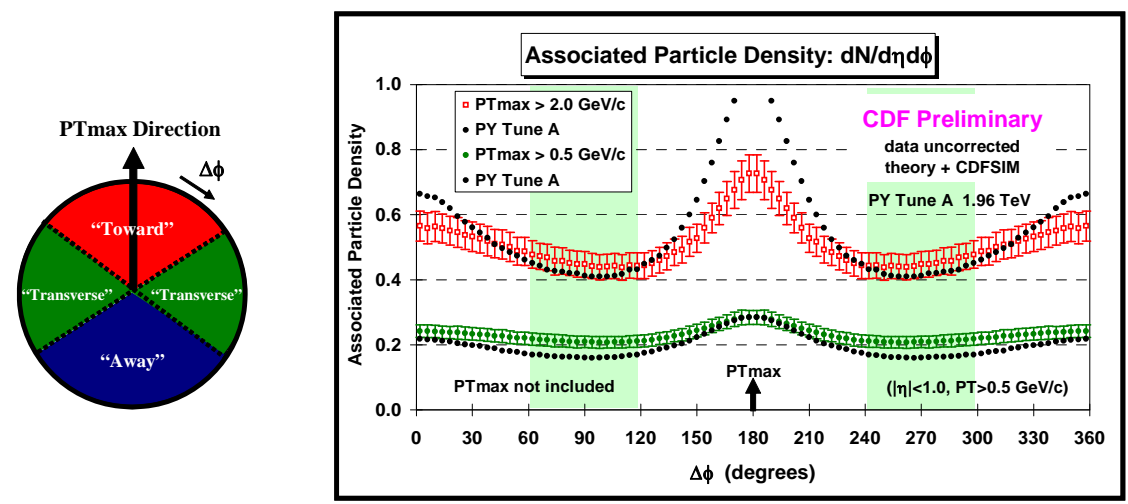

FIGURE 5. CDF Run 2 data on the $\Delta \phi$ dependence of the "associated" density of charged particles, $\mathrm{dN} / \mathrm{d} \eta \mathrm{d} \phi$, with $\mathrm{p}_{\mathrm{T}}>0.5 \mathrm{GeV} / \mathrm{c}$ and $|\eta|<1$ (not including PTmax) relative to the direction of PTmax (rotated to $180^{\circ}$ ) with PTmax $>0.5 \mathrm{GeV} / \mathrm{c}$ and PT $\max >2.0 \mathrm{GeV} / \mathrm{c}$ for "min-bias" collisions at $1.96 \mathrm{TeV}$ compared with PYTHIA Tune A. The "transverse" region is shaded. The plot shows (uncorrected) data compared with PYTHIA Tune A (after CDFSIM).

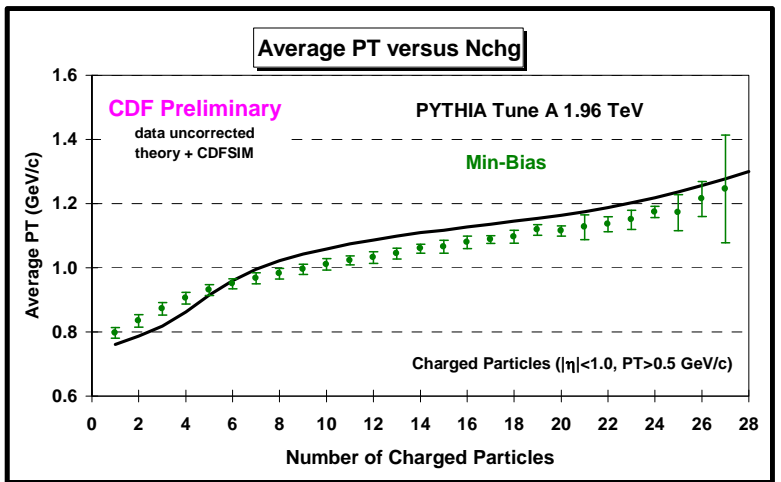

FIGURE 6. CDF Run 2 data on the average transverse momentum of charged particles versus the number of charged particles for the region $\left(\mathrm{p}_{\mathrm{T}}>0.5 \mathrm{GeV} / \mathrm{c},|\eta|<1\right)$ for "min-bias" collisions at 1.96 $\mathrm{TeV}$ compared with PYTHIA Tune A. The plot shows (uncorrected) data compared with PYTHIA Tune A (after CDFSIM).

Another observable that measures the relative amount of "hard" versus "soft" interactions in "min-bias" collisions is shown in Fig. 6. The CDF "min-bias" data show a rise in the average transverse momentum of charged particles as the number of charged particles increases. To get this correlation one must have a mixture of "soft" 
interactions with low multiplicity and "hard" interactions with higher multiplicity. Selecting a large multiplicity selects the "hard" processes resulting in a larger $\left\langle\mathrm{p}_{\mathrm{T}}\right\rangle$. This is not easy to reproduce and depends on your model of multiple parton interactions [10]. For example, if all multiple parton interactions were the same and independent, then selecting large multiplicity would select "min-bias" collisions with more multiple parton interactions, but $\left\langle\mathrm{p}_{\mathrm{T}}\right\rangle$ would not increase.

Unfortunately, one cannot easily extrapolate what we have learned about "minbias" collisions at the Tevatron to the LHC. One cannot trust the modeling of the energy dependence of multiple-parton interactions. Also, what one will see at the LHC will depend what "min-bias" trigger is used. Fig. 3 and Fig. 7 shows predictions of PYTHIA Tune A at $14 \mathrm{TeV}$. PYTHIA Tune A predicts a $42 \%$ rise in charged particle density, $\mathrm{dN} / \mathrm{d} \eta \mathrm{d} \phi$, at $\eta=0$ in going from the Tevatron $(1.8 \mathrm{TeV})$ to the $\mathrm{LHC}$ $(14 \mathrm{TeV})$. The 4 charged particles per unit $\eta$ seen in Fig. 2 at $1.8 \mathrm{TeV}$ become about 6 charged particles per unit $\eta$ at the LHC (for a CDF like trigger!). At the LHC a larger portion of the hard core cross section, $\sigma_{\mathrm{HC}}$, will be "hard". Tune A predicts that at 1.8 $\mathrm{TeV}$ about $1 \%$ of the hard core cross section involves hard parton-parton collisions with transverse momentum greater than $10 \mathrm{GeV} / \mathrm{c}$, which increases to $12 \%$ at the LHC. "Min-bias" at the LHC has more hard scattering and is more perturbative!
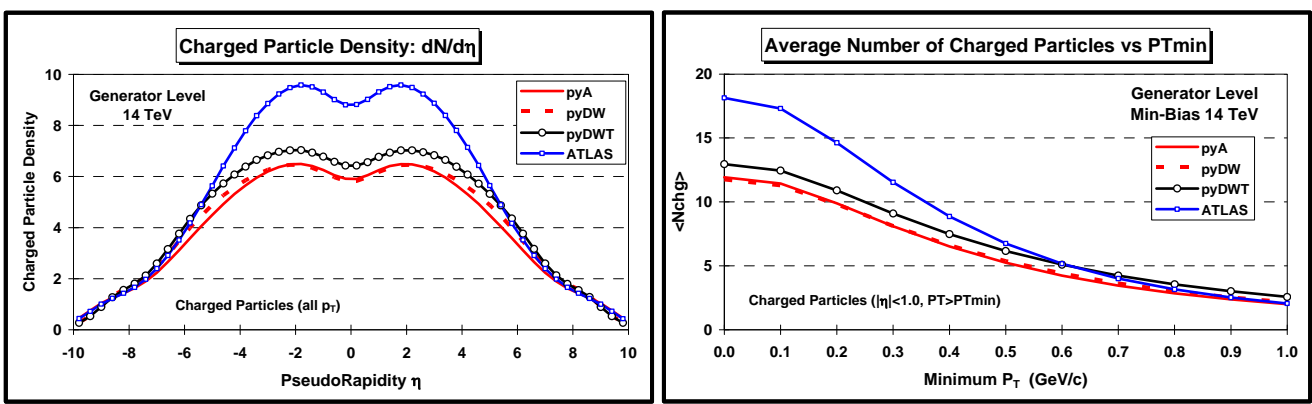

FIGURE 7. (left) Predictions for the "min-bias" (hard core component) charged particle density, $\mathrm{dN} / \mathrm{d} \eta$, for all $\mathrm{p}_{\mathrm{T}}$ at $14 \mathrm{TeV}$ from PYTHIA Tune A, Tune DW, Tune DWT, and the ATLAS PYTHIA tune. (right) Shows the average number of charged particles in the central region, $|\eta|<1$, as a function of the minimum observed $\mathrm{pT}$ for "min-bias" collisions (hard core component) at $14 \mathrm{TeV}$ predicted by PYTHIA Tune A, Tune DW, Tune DWT, and the ATLAS PYTHIA tune.

Fig. 7 shows predictions from several PYTHIA 6.2 tunes [11] for "min-bias" (hard core component) at the LHC. PYTHIA Tune DW is very similar to Tune A except $\operatorname{PARP}(67)=2.5$, which is the preferred value determined by DØ in fitting their dijet $\Delta \phi$ distribution [12]. PARP(67) sets the high $\mathrm{p}_{\mathrm{T}}$ scale for initial-state radiation in PYTHIA. It determines the maximal parton virtuality allowed in time-like showers. Tune DW and Tune DWT are identical at $1.96 \mathrm{TeV}$, but Tune DW and DWT extrapolate differently to the LHC. Tune DWT uses the ATLAS tune energy dependence, $\operatorname{PARP}(90)=0.16$, while Tune DW uses the Tune A value of PARP $(90)=$ 0.25. The CDF Tunes (Tune A, Tune DW, and Tune DWT) are very similar and predict about 6-7 charged particles per unit $\eta$ at $\eta=0$. The ATLAS tune [13] is quite different and predicts about 9 charged particles. As can be seen in Fig. 8, the ATLAS tune has a much softer $\mathrm{p}_{\mathrm{T}}$ distribution than the CDF tunes. We do not know what to expect at the LHC. For now I prefer PYTHIA Tune DW or Tune DWT over the 
ATLAS Tune because these tunes fit the CDF Run 2 "min-bias" and "underlying event" data much better than the ATLAS Tune.

Remember what one will see at the LHC will depend on the "min-bias" trigger. Fig. 8 shows the prediction for "min-bias" charged particle density, $d N / d \eta$, for all $\mathrm{p}_{\mathrm{T}}$ at $14 \mathrm{TeV}$ from PYTHIA Tune DWT. The individual contributions from single diffraction, double diffraction, and the "hard core" components are shown together with the sum of all three weighted by their cross sections (with no trigger). A trigger that accepts predominately "hard core" with very little single and double diffraction (like the CDF "min-bias" trigger) will see the "hard core" value of about 6.5 charged particles per unit $\eta$ at $\eta=0$. A trigger that accepts all three (with no bias) would see only about 5 charged particles per unit $\eta$ since single and double diffraction do not contribute many particles at $\eta=0$. The ideal situation is to have several "min-bias" or "low-bias" triggers. Different triggers select differing amounts of the components of the cross section and having more than one trigger allows one to better separate the individual contributions.

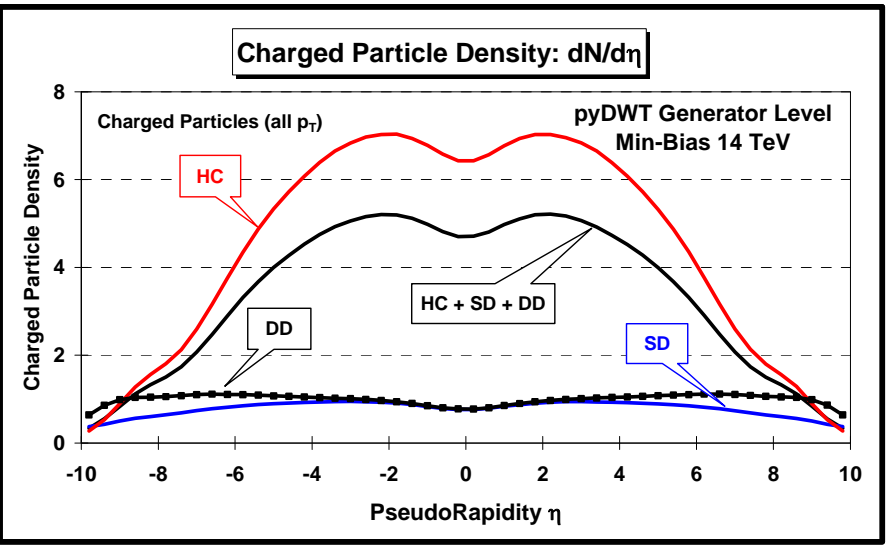

FIGURE 8. Predictions for the "min-bias" charged particle density, $d N / d \eta$, for all $\mathrm{p}_{\mathrm{T}}$ at $14 \mathrm{TeV}$ from PYTHIA Tune DWT. The individual contributions from single diffraction, double diffraction, and the "hard core" components are shown together with the sum of all three weighted by their cross sections (with no trigger).

The high luminosity at the LHC results in many proton-proton collisions per beam crossing. These extra collisions that come along in addition to the interaction that satisfied the trigger are referred to a "pile-up". Pile-up constitutes a new type of "minbias" trigger and one might be able to study the pile-up interactions as a way of studying "min-bias". The question is are the pile-up interactions unbiased? Well the answer depends on the primary trigger. If the extra pile-up interactions can help satisfy the trigger requirement, then they will and they will be biased. For example, a high $\mathrm{p}_{\mathrm{T}}$ jet trigger biases one toward more pile-up interactions and pile-up interactions that are more active. These pile-up interactions all transverse $\mathrm{p}_{\mathrm{T}}$ to the jets produced in the primary interaction and boost up their $\mathrm{p}_{\mathrm{T}}$ to satisfy the trigger requirement. The luminosity is now sufficiently high that one can study pile-up at the Tevatron. At CDF I am currently studying the pile-up interactions that accompany high $\mathrm{p}_{\mathrm{T}}$ jets and comparing it to the pile up accompanying "min-bias" collisions and high $\mathrm{p}_{\mathrm{T}}$ lepton pair production. For Drell-Yan lepton pair production pile-up cannot help satisfy the 
trigger and hence should be unbiased. However, even here one must be careful because the lepton isolation requirements bias one in favor of less active pile-up. I hope to learn a lot about pile-up at the Tevatron before we start taking data at the LHC.

\section{REFERENCES}

1. Measurement of the Proton-Antiproton Total Cross Section at 546 and 1,800 GeV, The CDF Collaboration (F. Abe et al.), Phys. Rev. D50, 5550-5561 (1994).

2. A Luminosity Independent Measurement of the Proton-Antiproton Total Cross Section at $1.8 \mathrm{TeV}$, N.A. Amos et al. (E-710 Collaboration), Phys. Lett. B243, 158-164 (1990).

3. Measurement of the Elastic, Total and Single Diffraction Cross-Sections at Tevatron Energies, S. Belforte (for the CDF Collaboration), Nuovo Cim. A107, 2085-2092 (1994).

4. Pseudorapidity Distributions of Charged Particles Produced in Proton-Antiproton Collisions at 630 and 1,800 GeV, F. Abe et al. (CDF Collaboration), Phys. Rev. D41, 2230 (1990).

5. Transverse Momentum Distributions of Charged Particles Produced in Proton-Antiproton Collisions at 630 and 1,800 GeV, F. Abe et al. (CDF Collaboration), Phys. Rev. Lett. 61, 1819 (1988).

6. T. Sjostrand, Phys. Lett. 157B, 321 (1985); M. Bengtsson, T. Sjostrand, and M. van Zijl, Z. Phys. C32, 67 (1986); T. Sjostrand and M. van Zijl, Phys. Rev. D36, 2019 (1987).

7. Min-Bias and the Underlying Event in Run 2 at CDF, R. Field, published in the proceedings of the XXXIV International Symposium on Multiparticle Dynamics, Acta Physica Polonica B36, 167-178, 2005.

8. G. Marchesini and B. R. Webber, Nucl. Phys. B310, 461 (1988); I. G. Knowles, Nucl. Phys. B310, 571 (1988); S. Catani, G. Marchesini, and B. R. Webber, Nucl. Phys. B349, 635 (1991).

9. Charged Jet Evolution and the Underlying Event in Proton-Antiproton Collisions at $1.8 \mathrm{TeV}$, The CDF Collaboration (T. Affolder et al.), Phys. Rev. D65, 092002 (2002).

10. Non-perturbative QCD Effects and the Top Mass at the Tevatron, P. Skands and D. Wicke, FERMILAB-PUB-06-340-T, 2007, hep-ph/0703081.

11. CDF Run 2 Monte-Carlo Tunes, R. Field, CDF/PUB/JET/PUBLIC/8547, Pubublished in the Proceedings of the TeV4LHC Workshop - 4th Meeting, Fermi National Accelerator Laboratory, Batavia, IL, October 20-22, 2005. FERMILAB-CONF-06-408-E.

12. Phys. Rev. Lett. 94, 221801 (2005).

13. Prediction for Minimum Bias and the Underlying Event at LHC Energies, A. Moraes, C. Buttar, and I. Dawson, Eur. Phys. J. C50, 435-466 (2007). 